\title{
Initial Results of a Silicon Sensor Irradiation Study for ILC Extreme Forward Calorimetry
}

\author{
Talk presented at the International Workshop on Future \\ Linear Colliders (LCWS13), Tokyo, Japan, 11-15 \\ November 2013.
}

\author{
Reyer Band ${ }^{\mathrm{a}}$, Vitaliy Fadeyev ${ }^{\mathrm{a}}$, R. Clive Field ${ }^{\mathrm{b}}$, Spencer Key ${ }^{\mathrm{a}}$, Tae Sung \\ Kim $^{\mathrm{a}}$, Thomas Markiewicz ${ }^{\mathrm{b}}$, Forest Martinez-McKinney ${ }^{\mathrm{a}}$, Takashi \\ Maruyama $^{\mathrm{b}}$, Khilesh Mistry ${ }^{\mathrm{a}}$, Ravi Nidumolu ${ }^{\mathrm{a}}$, Bruce A. Schumm ${ }^{\mathrm{a}, *}$, Edwin \\ Spencer $^{\mathrm{a}}$, Conor Timlin ${ }^{\mathrm{a}}$, Max Wilder ${ }^{\mathrm{a}}$ \\ ${ }^{a}$ Santa Cruz Institute for Particle Physics and the University Of California, 1156 High \\ Street, Santa Cruz California 95064 USA \\ ${ }^{b}$ SLAC National Accelerator Laboratory, 2575 Sand Hill Road, Menlo Park California \\ 94025 USA
}

\begin{abstract}
Detectors proposed for the International Linear Collider (ILC) incorporate a tungsten sampling calorimeter ('BeamCal') intended to reconstruct showers of electrons, positrons and photons that emerge from the interaction point of the collider with angles between 5 and 50 milliradians. For the innermost radius of this calorimeter, radiation doses at shower-max are expected to reach 100 MRad per year, primarily due to minimum-ionizing electrons and positrons that arise in the induced electromagnetic showers of e+e- 'beamstrahlung' pairs produced in the ILC beam-beam interaction. However, radiation damage to calorimeter sensors may be dominated by hadrons induced by nuclear interactions of shower photons, which are much more likely to contribute to the non-ionizing energy loss that has been observed to damage sensors exposed to hadronic radiation. We report here on the results of SLAC Experiment T-506, for which several different types of silicon diode sensors
\end{abstract}

\footnotetext{
${ }^{*}$ Corresponding author.

Email address: baschumm@ucsc.edu (Bruce A. Schumm )
} 
were exposed to doses of radiation induced by showering electrons of energy 3.5-10.6 GeV. By embedding the sensor under irradiation within a tungsten radiator, the exposure incorporated hadronic species that would potentially contribute to the degradation of a sensor mounted in a precision sampling calorimeter. Depending on sensor technology, efficient charge collection was observed for doses as large as 220 MRad.

Keywords: radiation damage, electromagnetic showers, silicon diode sensors, sampling calorimetry

\section{Introduction}

Far-forward calorimetry, covering the region between 5 and 50 milliradians from the on-energy beam axis, is envisioned as a component of both the ILD [1] and SiD [2] detector concepts for the proposed International Linear Collider (ILC). The BeamCal tungsten sampling calorimeter proposed to cover this angular region is expected to absorb approximately $10 \mathrm{TeV}$ of electromagnetic radiation per beam crossing from e+e- beamstrahlung pairs, leading to expected annual radiation doses of $100 \mathrm{MRad}$ for the most heavily-irradiated portions of the instrument. While the deposited energy is expected to arise primarily from minimum-ionizing electrons and positrons in the induced electromagnetic showers, radiation damage to calorimeter sensors may be dominated by hadrons induced by nuclear interactions of shower photons, which are much more likely to contribute to the non-ionizing energy loss that has been observed to damage sensors exposed to hadronic radiation. We report here on the results of SLAC Experiment T-506, for which several different types of silicon diode sensors were exposed to doses of up to $220 \mathrm{MRad}$ at the approximate maxima of electromagnetic showers induced in a tungsten radiator by electrons of energy $3.5-10.6 \mathrm{GeV}$, similar to that of electrons and positrons from ILC beamstrahling pairs.

Bulk damage leading to the suppression of the electron/hole chargecollection efficiency is generally thought to be proportional to the non-ionizing energy loss ('NIEL') component of the energy deposited by the incident radiation. Early studies of electromagnetically-induced damage to solar cells [3, 4, 5] suggested that p-type bulk sensors were more tolerant to damage from electromagnetic sources, due to an apparent departure from NIEL scaling, particularly for electromagnetic particles of lower incident energy.

Several more-recent studies have explored radiation tolerance to incident 
fluxes of electrons. A study assessing the capacitance vs. bias voltage (CV) characteristics of sensors exposed to as much as 1 GRad of incident $2 \mathrm{MeV}$ electrons [6] suggested approximately 35 times less damage to n-type magnetic Czochralski sensors than that expected from NIEL scaling. A study of various n-type sensor types exposed to $900 \mathrm{MeV}$ electrons showed chargecollection loss of as little as 3\% for exposures up to 50 MRad exposure [7]; for exposures of $150 \mathrm{MRad}$, a suppression of damage relative to NIEL expectations of up to a factor of four was observed [8]. These discrepancies have been attributed to the different types of defects created by lattice interactions: electrons tend to create point-like defects that are more benign than the clusters formed due to hadronic interactions.

Finally, in studies of sensors exposed to large doses of hadron-induced radiation, p-type bulk silicon was found to be more radiation-tolerant than n-type bulk silicon, an observation that has been attributed to the absence of type inversion and the collection of an electron-based signal [9, 10]. However, n-type bulk devices have certain advantages, such as a natural inter-electrode isolation with commonly used passivation materials such as silicon oxide and silicon nitride.

Here, we report on an exploration of the radiation tolerance of silicon sensors, assessed via direct measurements of the median collected charge deposited by minimum-ionizing particles, for four different bulk compositions: p-type and n-type doping of both magnetic Czochralski and float-zone crystals. The p-type float-zone sensors were produced by Hamamatsu Photonics while the remaining types were produced by Micron Corporation. Sensor strip pitch varied between 50 and $100 \mu \mathrm{m}$, while the bulk thickness varied between $307 \mu \mathrm{m}$ (for the p-type magnetic Czochralski sensors) and $320 \mu \mathrm{m}$ (for the p-type float zone sensors). The use of these sensors is being explored as an alternative to several more novel sensor technologies that are currently under development [1], including GaAs and CVD diamond.

While the radiation dose was initiated by electromagnetic processes (electrons showering in tungsten), the placement of the sensors near shower max ensures that the shower incorporates an appropriate component of hadronic irradiation arising from neutron spallation, photoproduction, and the excitation of the $\Delta$ resonance. Particularly for the case that NIEL scaling suppresses electromagnetically-induced radiation damage, the small hadronic component of the electromagnetic shower might dominate the rate of damage to the sensor. However, the size and effect of this component is difficult to estimate reliably, and so we choose to study radiation damage in a configura- 
tion that naturally incorporates all components present in an electromagnetic shower.

\section{Experimental Setup}

Un-irradiated sensors were subjected to current vs. bias voltage (IV) and $\mathrm{CV}$ tests, the results of which allowed a subset of them to be selected for irradiation based on their breakdown voltage (typically above $1000 \mathrm{~V}$ for selected sensors) and low level of leakage current. The sensors were placed on carrier printed-circuit 'daughter boards' and wire-bonded to a readout connector. The material of the daughter boards was milled away in the region to be irradiated in order to facilitate the charge collection measurement (described below) and minimize radio-activation. The median collected charge was measured with the Santa Cruz Institute for Particle Physics (SCIPP) charge-collection (CC) apparatus (also described below) before irradiation. The sensors remained mounted to their individual daughter boards throughout irradiation and the followup tests, simplifying their handling and reducing uncontrolled annealing. Additionally, this allowed a reverse-bias voltage to be maintained across the sensor during irradiation. The voltage was kept small (at the level of a few volts) to avoid possible damage of the devices from a large instantaneous charge during the spill.

Sensors were irradiated with beam provided by the End Station Test Beam (ESTB) facility at the SLAC National Accelerator Laboratory. Parameters of the beam provided by the ESTB facility are shown in Table 1. The beam was incident upon a series of tungsten radiators, as enumerated in Table 2. An initial $7 \mathrm{~mm}$-thick tungsten plate served to initiate the electromagnetic shower. The small number of radiation lengths of this initial radiator (2.0) permitted the development of a small amount of divergence of the shower relative to the straight-ahead beam direction without significant development of the largely isotropic hadronic component of the shower.

This plate was followed by an open length of approximately $55 \mathrm{~cm}$, which allowed a degree of spreading of the shower before it impinged upon a second, significantly thicker radiator (4.0 radiation lengths) which was followed immediately by the sensor undergoing irradiation. This was closely followed, in turn, by an 8.0 radiation-length radiator. Immediately surrounding the sensor by tungsten radiators that both initiated and absorbed the great majority of the electromagnetic shower ensured that the sensor would be illuminated 
Table 1: Parameters of the beam delivered by the ESTB facility during the T-506 experiment.

\begin{tabular}{cc} 
Parameter & Value \\
\hline Energy & $3.5-10.6 \mathrm{GeV}$ \\
Repetition Rate & $5-10 \mathrm{~Hz}$ \\
Charge per Pulse & $150 \mathrm{pC}$ \\
Spot Size (radius) & $\sim 1 \mathrm{~mm}$
\end{tabular}

by a flux of hadrons commensurate with that experienced by a calorimeter sensor close to the maximum of a tungsten-induced shower.

Table 2: Elements encountered by incident beam as a function of longitudinal position, relative to the upstream face of the initial tungsten radiator.

\begin{tabular}{lcc} 
Element & $\begin{array}{c}\text { Longitudinal } \\
\text { Position }(\mathrm{cm})\end{array}$ & $\begin{array}{c}\text { Accumulated } \\
\text { Radiation Lengths }\end{array}$ \\
\hline Front face radiator 1 & 0.0 & 0.0 \\
Back face radiator 1 & 0.7 & 2.0 \\
Front face radiator 2 & 55.0 & 2.0 \\
Back face radiator 2 & 55.7 & 4.0 \\
Front face radiator 3 & 56.3 & 4.0 \\
Back face radiator 3 & 57.0 & 6.0 \\
Sensor sample & 57.7 & 6.0 \\
Front face radiator 4 & 59.0 & 6.0 \\
Back face radiator 4 & 60.4 & 10.0 \\
Front face radiator 5 & 61.0 & 10.0 \\
Back face radiator 5 & 62.4 & 14.0
\end{tabular}

Although initiating the shower significantly upstream of the sensor promoted a more even illumination of the sensor than would otherwise have been achieved, the half-width of the resulting electron-positron fluence distribution at the sensor plane was less than $0.5 \mathrm{~cm}$. On the other hand, the aperture of the CC apparatus (to be described below) was of order $0.7 \mathrm{~cm}$. Thus, in order to ensure that the radiation dose was well understood over the region of exposure to the $\mathrm{CC}$ apparatus source, it was necessary to achieve a uniform illumination over a region of approximately $1 \mathrm{~cm}^{2}$. This was done by 
'rastering' the detector across the beam spot through a range of $1 \mathrm{~cm}$ in the directions both along and transverse to the direction of the sensor's strips, generating a region of approximately $1 \mathrm{~cm}^{2}$ over which the illumination was uniform to within $\pm 5 \%$.

\section{Dose Rates}

During the $120 \mathrm{~Hz}$ operation of the SLAC Linear Collider Light Source (LCLS), 5-10 Hz of beam was deflected by a pulsed kicker magnet into the End Station transfer line. The LCLS beam was very stable with respect to both current and energy. Electronic pickups and ion chambers measured the beam current and beam loss through the transfer line aperture, ensuring that good transfer efficiency could be established and maintained. The transfer efficiency was estimated to be $(95 \pm 5) \%$, although for the highest energy beams delivered in the final days of T-506, the transfer line experienced small but persistent beam loss; for this period, the transfer efficiency was measured to be $(90 \pm 10) \%$. These transfer factors and their uncertainties were taken into account in the estimation of dose rates through the exposed sensors.

To calculate the dose rate through the sensor, it is necessary to determine the 'shower conversion factor' $\alpha$ that provides the mean fluence of minimumionizing particles (predominantly electrons and positrons), in particles per $\mathrm{cm}^{2}$, per incoming beam electron. This factor is dependent upon the radiator configuration and incident beam energy, as well as the rastering pattern used to provide an even fluence across the sensor (as stated above, the detector was translated continuously across the beam centerline in a $1 \mathrm{~cm}^{2}$ square pattern).

To estimate $\alpha$, the Electron-Gamma-Shower (EGS) Monte Carlo program [11] was used to simulate showers through the radiator configuration and into the sensor. The configuration of Table 2 was input to the EGS program, and a mean fluence profile (particles per $\mathrm{cm}^{2}$ through the sensor as a function of transverse distance from the nominal beam trajectory) was accumulated by simulating the showers of 1000 incident electrons of a given energy. To simulate the rastering process, the center of the simulated profile was then moved across the face of the sensor in $0.5 \mathrm{~mm}$ steps, and an estimated mean fluence per incident electron as a function of position on the sensor (again, relative to the nominal beam trajectory) was calculated. This resulted in a mean fluence per incident electron that was uniform to within 
a few percent $1 \mathrm{~mm}$ or more inside of the edge of the rastering region. The value of $\alpha$ used for subsequent irradiation dose estimates was taken to be the value found at the intersection of the nominal beam trajectory with the sensor plane. The simulation was repeated for various values of the incident electron energy, producing the values of $\alpha$ shown in Table 3 .

Table 3: Shower conversion factor $\alpha$, giving the mean fluence at the sensor per incident electron, as a function of electron energy. These values include the effect of rastering over a $1 \mathrm{~cm}^{2}$ area surrounding the nominal beam trajectory. Also shown is the number of Rads per $\mathrm{nC}$ of delivered charge, at the given energy, corresponding to the given value of $\alpha$.

\begin{tabular}{lcc}
$\begin{array}{l}\text { Electron } \\
\text { Energy }(\mathrm{GeV})\end{array}$ & $\begin{array}{c}\text { Shower Conversion } \\
\text { Factor } \alpha\end{array}$ & $\begin{array}{c}\text { Dose per nC Delivered } \\
\text { Charge }(\mathrm{kRad})\end{array}$ \\
\hline 2 & 2.1 & 0.34 \\
4 & 9.4 & 1.50 \\
6 & 16.5 & 2.64 \\
8 & 23.5 & 3.76 \\
10 & 30.2 & 4.83 \\
12 & 36.8 & 5.89
\end{tabular}

To convert this number to Rads per nC of delivered charge, a mean energy loss in silicon of $3.7 \mathrm{MeV} / \mathrm{cm}$ was assumed, leading to a fluence-to-Rad conversion factor of $160 \mathrm{Rad}$ per $\mathrm{nC} / \mathrm{cm}^{2}$. It should be noted that, while this dose rate considers only the contribution from electrons and positrons, these two sources dominate the overall energy absorbed by the sensor. In addition, the BeamCal dose-rate spec of 100 MRad per year considered only the contribution from electrons and positrons.

In order to accurately estimate the dose rates, it was also necessary to ensure that the nominal beam trajectory passed through a well-known and reproducible position on the sensors. A jig attached to the downstream side of Radiator 3 (see Table 2) positioned the daughter board carrying the sensor at a fixed position relative to the radiator configuration. Each sensor was mounted onto its own daughter board at a location reproducible to submillimeter accuracy. The desired location of the nominal beam trajectory in the middle of the $1 \mathrm{~cm}^{2}$ rastering pattern was then transfered to the upstream face of Radiator 2, which was rigidly attached to Radiator 3, using a mechanical metrology procedure. A Delrin pin was attached to the upstream face of Radiator 2 at a known displacement from the desired beam location, 
which was then used to spindle a reticled phosphorescent screen. The sensor/radiator assembly was then moved to the center of the rastering pattern, and with Radiator 1 removed, the beam was steered until it hit the intended place on the reticled screen. With the beam trajectory thus established, Radiator 1 was replaced and two upstream phosphorescent screens were placed in the beamline. The position of the beam on these screens was recorded, establishing both the position and angle of the properly steered beam.

To confirm the adequacy of the dose-calibration simulation (described above) and this alignment procedure, an in-situ measurement of the dose was made using a radiation-sensing field-effect transistor ('RADFET') [12] positioned on a daughter board at the expected position of the nominal beam trajectory at the center of the rastering pattern. Beam was delivered in $150 \mathrm{pC}$ pulses of $4.02 \mathrm{GeV}$ electrons; a total of 1160 pulses were directed into the target over a period of four minutes, during which the sensor was rastered quickly through its $1 \mathrm{~cm}^{2}$ pattern. The RADFET was then read out, indicating a total accumulated dose of $230 \mathrm{kRad}$, with an uncertainty of roughly $10 \%$. Making use of the dose rate calibration of Table 3 , interpolating to the exact incident energy of $4.02 \mathrm{GeV}$, and taking into account the $(95 \pm$ $5) \%$ transfer efficiency of the ESTB beamline, leads to an expected dose of 250 kRad, within the $\sim 10 \%$ uncertainty of the RADFET measurement.

\section{Sensor Irradiation Levels}

As mentioned above, four types of sensors were studied: p-type and ntype doped versions of both magnetic Czochralski and float-zone crystals. In what follows, we will use the notation ' $\mathrm{N}$ ' ('P') for n-type (p-type) bulk sensors, and 'F' ('C') for float-zone (magnetic Czochralski) crystal technology. Once a sensor was irradiated with the ESTB, it was placed in a sub-freezing environment and not irradiated again. Up to four sensors of each type were irradiated and chilled until they could be brought back to the University of California, Santa Cruz campus for the post-irradiation CC measurement. In addition, the sub-freezing environment was maintained both during and after the CC measurement, so that controlled annealing studies can eventually be done.

Table 4 displays the dose parameters of the irradiated sensors. The $(95 \pm$ $5) \%$ transfer line efficiency has been taken into account in these estimates. The numeral following the two letters in the sensor identifier refer to an arbitrary ordering of sensors assigned during the sensor selection. Sensors 
were held at between 0 and $5 \mathrm{C}$ during irradiation. With the exception of sensor $\mathrm{NC} 02$, which was accidentally annealed for 5 hours at temperatures as high as $130 \mathrm{C}$, all sensors were transferred to a cold (below -10 C) environment immediately after irradiation. All four sensor types were exposed to dose rates of approximately 5 and $20 \mathrm{MRad}$, while an NF sensor received over 90 MRad and an NC sensor 220 MRad. CC results for the irradiated sensors will be presented below.

Table 4: Dose parameters of the irradiated sensors. The $(95 \pm 5) \%$ transfer line efficiency has been taken into account in these estimates. While the NC02 sensor was irradiated at a temperature of $5 \mathrm{C}$, it was accidentally annealed for approximately 5 hours at temperatures as high as $130 \mathrm{C}$. Also included is the minimum voltage for full depletion $\left(V_{F D}\right)$ for the sensors before irradiation.

\begin{tabular}{cccccc} 
Sensor & $V_{F D}$ & $\begin{array}{c}\text { Irradiation } \\
\text { Temp. }(\mathrm{C})\end{array}$ & $\begin{array}{c}\text { Beam Energy } \\
(\mathrm{GeV})\end{array}$ & $\begin{array}{c}\text { Delivered } \\
\text { Charge }(\mu \mathrm{C})\end{array}$ & $\begin{array}{c}\text { Dose } \\
(\mathrm{MRad})\end{array}$ \\
\hline \hline PF05 & 190 & 0 & 5.88 & 2.00 & 5.13 \\
PF14 & 190 & 0 & 3.48 & 16.4 & 19.7 \\
\hline PC10 & 660 & 0 & 5.88 & 1.99 & 5.12 \\
PC08 & 700 & 0 & $(5.88,4.11,4.18)$ & $(3.82,3.33,3.29)$ & 20.3 \\
\hline NF01 & 90 & 0 & 4.18 & 2.30 & 3.68 \\
NF02 & 90 & 0 & 4.02 & 12.6 & 19.0 \\
NF07 & 100 & 5 & 8.20 & 23.6 & 91.4 \\
\hline NC01 & 220 & 0 & 5.88 & 2.00 & 5.13 \\
NC10 & 220 & 0 & 3.48 & 15.1 & 18.0 \\
NC03 & 220 & 5 & 4.01 & 59.9 & 90.2 \\
NC02 & 220 & $5^{*}$ & $(10.60,8.20)$ & $(32.3,13.8)$ & 220 \\
\hline
\end{tabular}

\section{Charge Collection Measurement}

The SCIPP CC apparatus incorporates a ${ }^{90} \mathrm{Sr}$ source that has a secondary $\beta$-decay with an end-point energy of $2.28 \mathrm{MeV}$. These $\beta$ particles illuminate the sensor under study, 64 channels of which are read out by the PMFE ASIC [13], with a shaping time of 300 nsec. Whenever one of the 64 channels exceeds a pre-set, adjustable threshold, the time and duration of the excursion over threshold is recorded. In addition, the $\sim 250 \mathrm{~Hz}$ of $\beta$ particles that pass through the sensor, and subsequently enter a small ( $2 \mathrm{~mm}$ horizontal by $7 \mathrm{~mm}$ vertical) slit, trigger a scintillator, and the time of excitation 
of the scintillator is also recorded. If the slit is properly aligned with the read-out channels of the sensor, and the sensor is efficient at the set read-out threshold, a temporal coincidence between the scintillator pulse and one of the read-out channels will be found in the data stream.

Coincidence Profile

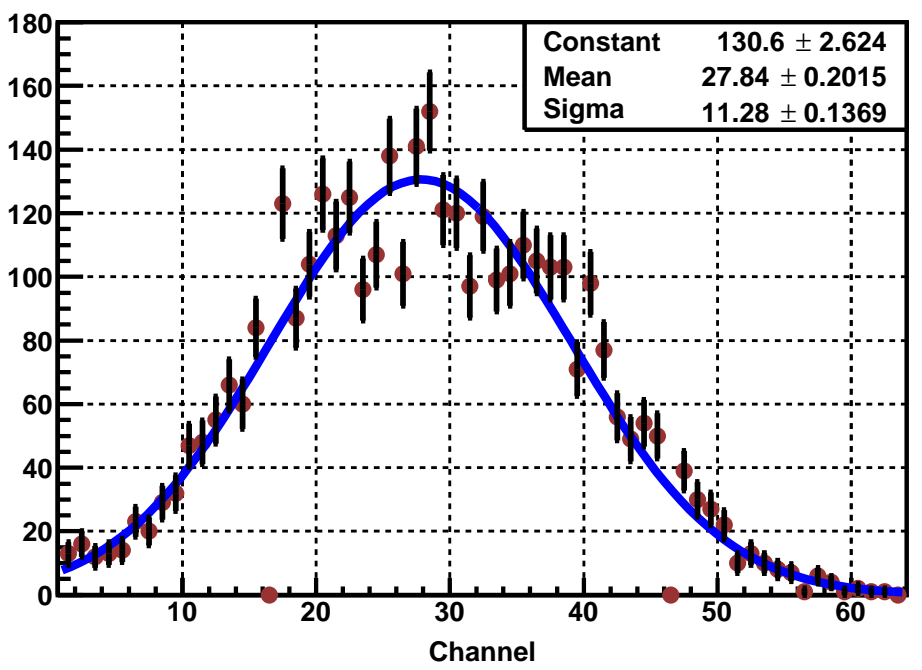

Figure 1: Sample profile of coincidences between the read-out sensor channels and the trigger scintillator. The integral of this distribution provides a count of the number of $\beta$ particles triggering the scintillator that also exceed the chosen PMFE threshold in one of the read-out channels.

Figure 1 shows a sample coincidence profile (histogram of the number of coincidences vs. channel number) for a 150-second run at a given threshold and reverse bias level for one of the irradiated sensors (specifically, for the NC01 sensor after 5.1 MRad of irradiation, applying a 300V reverse bias and a $130 \mathrm{mV}$ threshold). The integral of the distribution yields an estimate of the total number of coincidences found during the run, which, when divided by the number of scintillator firings (after a small correction for cosmic background events) yields the median CC level at that threshold and bias level. This measurement can then be performed as a function of threshold level, yielding the curve shown in Figure 2. For this plot, the abscissa has been converted from voltage (the applied threshold level) to fC (the PMFE input charge that will fire the threshold with exactly $50 \%$ efficiency) via a prior calibration step involving measurement of the PMFE response to known values of injected charge. The point at which the curve in Figure 2 crosses the 
$50 \%$ level yields the median $\mathrm{CC}$ for the given bias level. In a prior study of sensors irradiated with hadrons, the SCIPP apparatus gave median charge results consistent with that of other charge collection systems used to assess radiation damage in that study [14].

Charge Collection Efficiency vs. Threshold: Bias $=200 \mathrm{~V}$

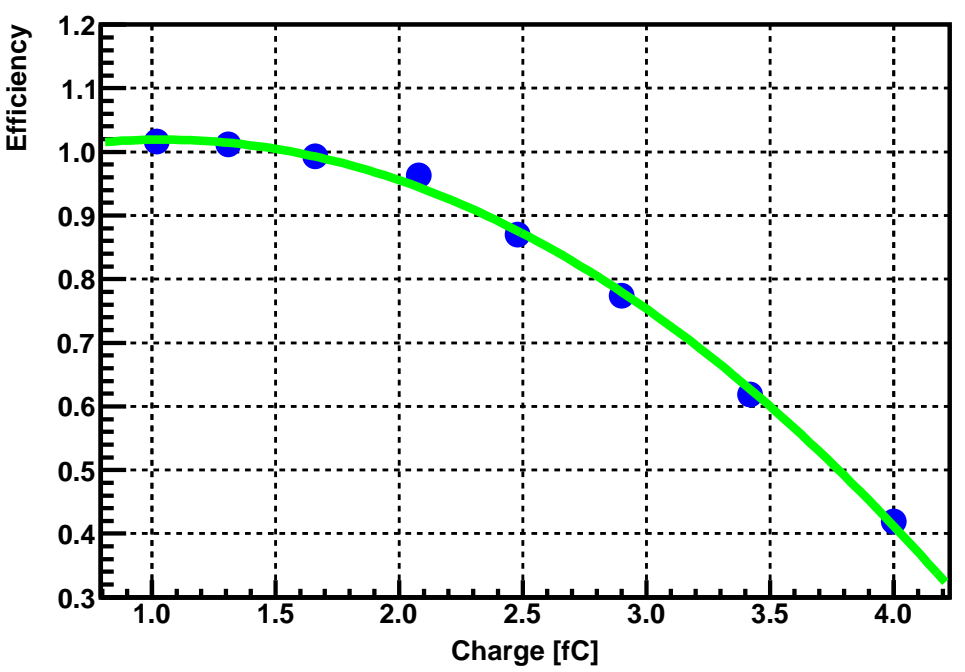

Figure 2: Plot of efficiency vs. PMFE threshold setting for one of the irradiated sensors. The abscissa has been converted from applied threshold voltage to the amount of input PMFE charge that will exceed the given threshold exactly $50 \%$ of the time. The point at which the curve crosses the $50 \%$ level yields the median CC for the given bias level.

\section{Charge Collection Results}

The daughter boards containing the irradiated sensors were designed with connectors that allowed them to be attached to the $\mathrm{CC}$ apparatus readout board without handling the sensors. The median $\mathrm{CC}$ was measured as a function of reverse bias voltage for each sensor both before and after irradiation.

The best performance was observed for the NC (n-type bulk magnetic Czochralski) sensor type. For the exposures of 5.1 (NC01) and 18.0 MRad (NC10), no difference in charge collection performance was observed relative to the pre-irradiation studies of the NC01 and NC10 sensors. In Figure 3 the median $\mathrm{CC}$ both before and after irradiation is plotted for the NC03 


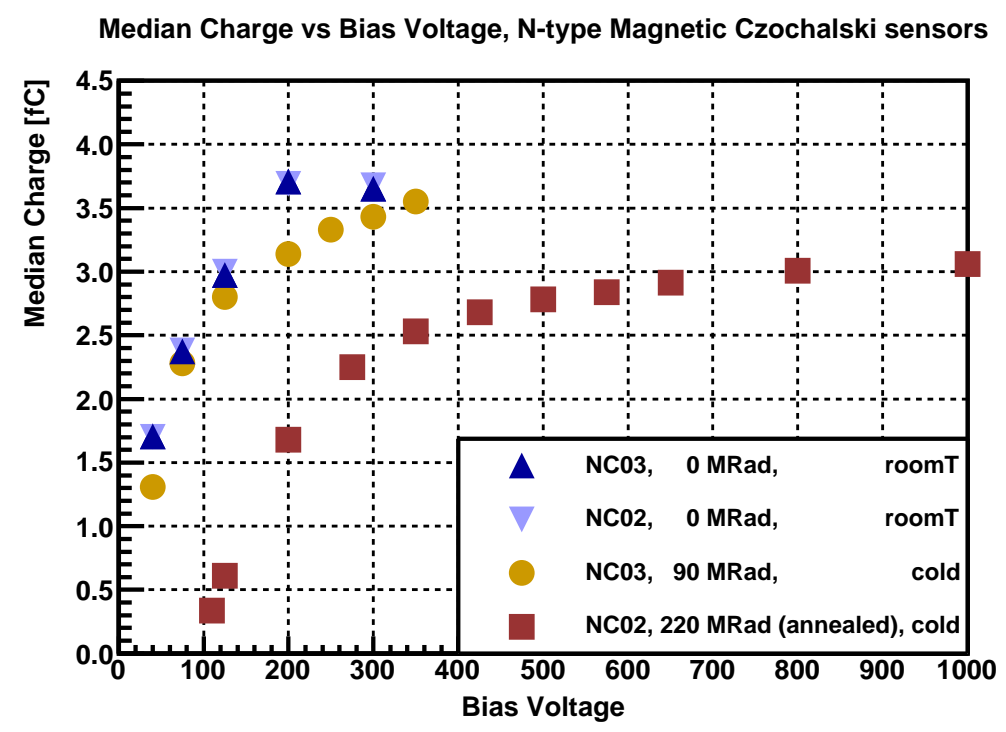

Figure 3: High-dose CC results for the NC03 (90 MRad dose) and NC02 (220 MRad dose) sensors.

(90 MRad dose) and NC02 (220 MRad dose) sensors; it should be borne in mind, though, that the $\mathrm{NC} 02$ sensor experienced significant annealing before the post-irradiation measurement was done. It is seen that, while the depletion voltage increases significantly with dose, median CC within $20 \%$ of un-irradiated values is maintained for doses above $200 \mathrm{MRad}$, although it may require annealing to maintain efficiency at that level.

Figures 4 through [6] show the results for the remaining three sensor types (PF, PC and NF) for irradiation levels up to approximately 20 MRad. Charge collection remains high for the $\mathrm{PC}$ and $\mathrm{NF}$ sensors at this dose level, with the PF sensors showing 10-20\% charge collection loss at 19.7 MRad. While this represents a dose of only about $20 \%$ of the expected annual dose for the most heavily-irradiated sensors in the BeamCal instrument, it is possible that a period of controlled annealing may restore some or all of the CC loss for these sensors. An NF sensor (NF07) with a 91 MRad exposure remains to be evaluated with the SCIPP CC apparatus.

Table 5 provides a table of maximum median collected charge, both before and after irradiation, and median charge loss due to irradiation. Not shown are results for the PC10 (damaged during handling) and NF07 (still under study) sensors. 


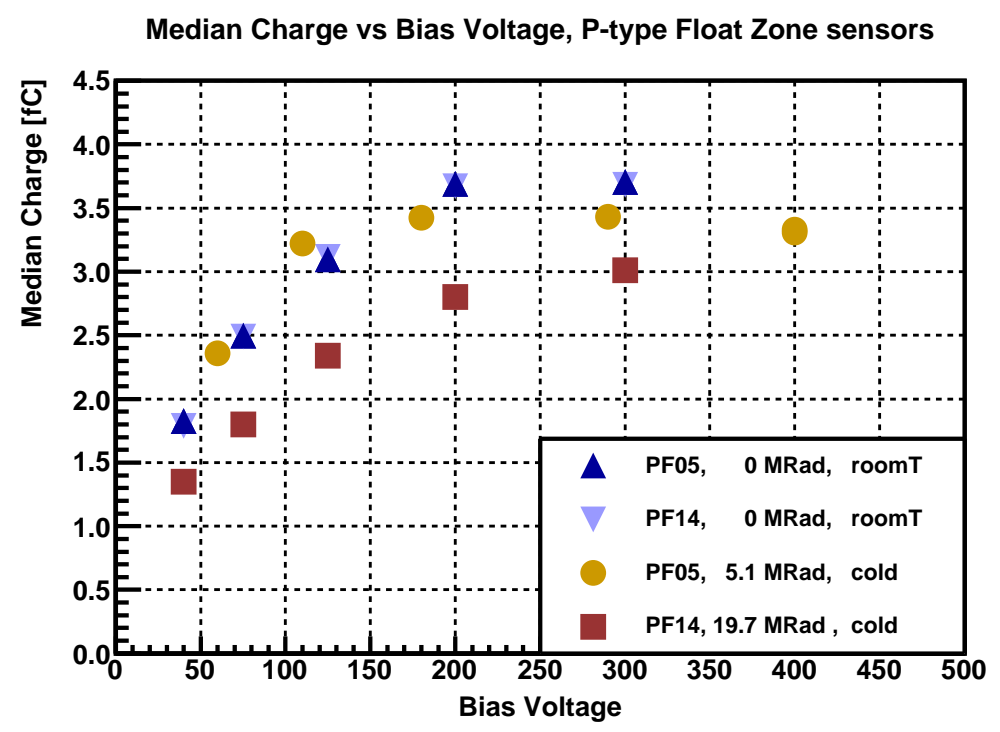

Figure 4: CC results for the PF05 (5.1 MRad dose) and PF14 (19.7 MRad dose) sensors.

\section{Summary and Conclusions}

We have explored the radiation tolerance of four different types of silicon diode sensors (n-type and p-type Float Zone and Magnetic Czochralski bulk sensors), exposing them to doses as high as 220 MRad at the approximate maxima of tungsten-induced electromagnetic showers. We have found all types to be radiation tolerant to $20 \mathrm{MRad}$, with the n-type Czochralski sensors exhibiting less than a $20 \%$ reduction in median collected charge for a dose in excess of 200 MRad. This suggests the possibility of charge collection sufficient for the operation of a calorimeter exposed to hundreds of MRad, approaching the specification required for the most heavily irradiated sensors in the ILC BeamCal instrument. We plan to follow through with IV and CV studies of the irradiated sensors, as well as annealing studies on selected sensors.

\section{Acknowledgments}

We are grateful to Leszek Zawiejski, INP, Krakow for supplying us with the tungsten plates needed to form our radiator. We also would like to express our gratitude to the SLAC Laboratory, and particularly the End Station 


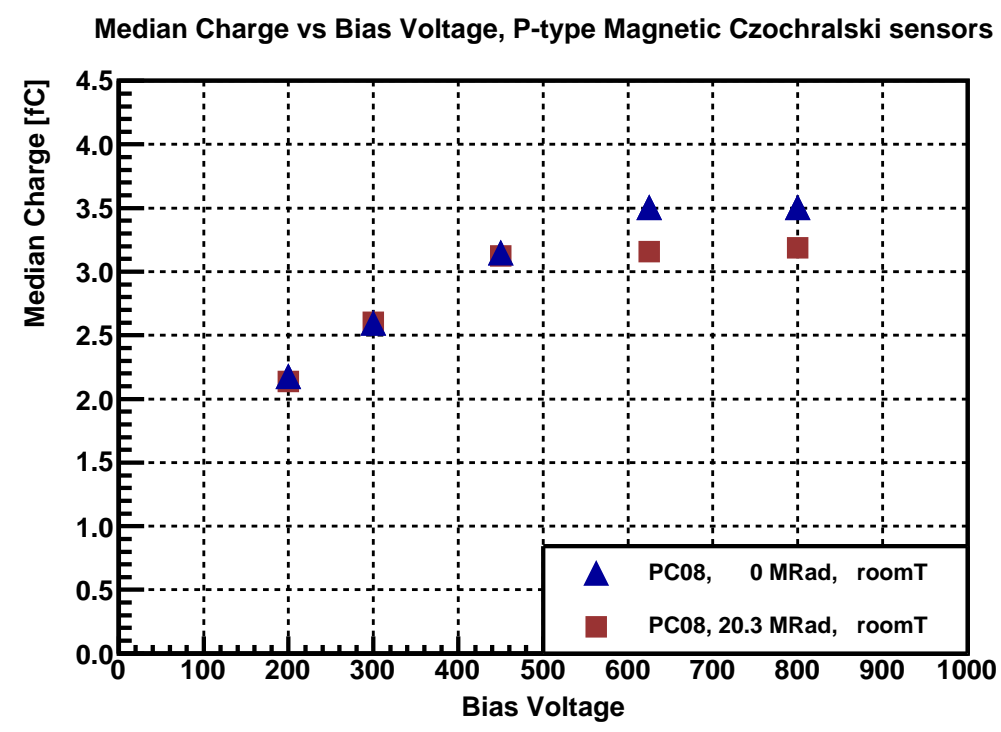

Figure 5: CC results for the PC08 (20.3 MRad dose) sensor. The PC10 sensor (5.1 MRad dose) suffered damage during handling and did not give reliable results.

Test Beam delivery and support personnel, who made the run possible and successful. Finally, we would like to thank our SCIPP colleague Hartmut Sadrozinski for the numerous helpful discussions and guidance he provided us.

\section{Role of the Funding Source}

The work described in this article was supported by the United States Department of Energy, DOE contract DE-AC02-7600515 (SLAC) and grant DE-FG02-04ER41286 (UCSC/SCIPP). The funding agency played no role in the design, execution, interpretation, or documentation of the work described herein.

\section{References}

[1] ILD Concept Group, International Large Detector DBD, http://www.linearcollider.org/ILC/physics-detectors/Detectors/

Detailed-Baseline-Design, Chapter 4 (2012). 


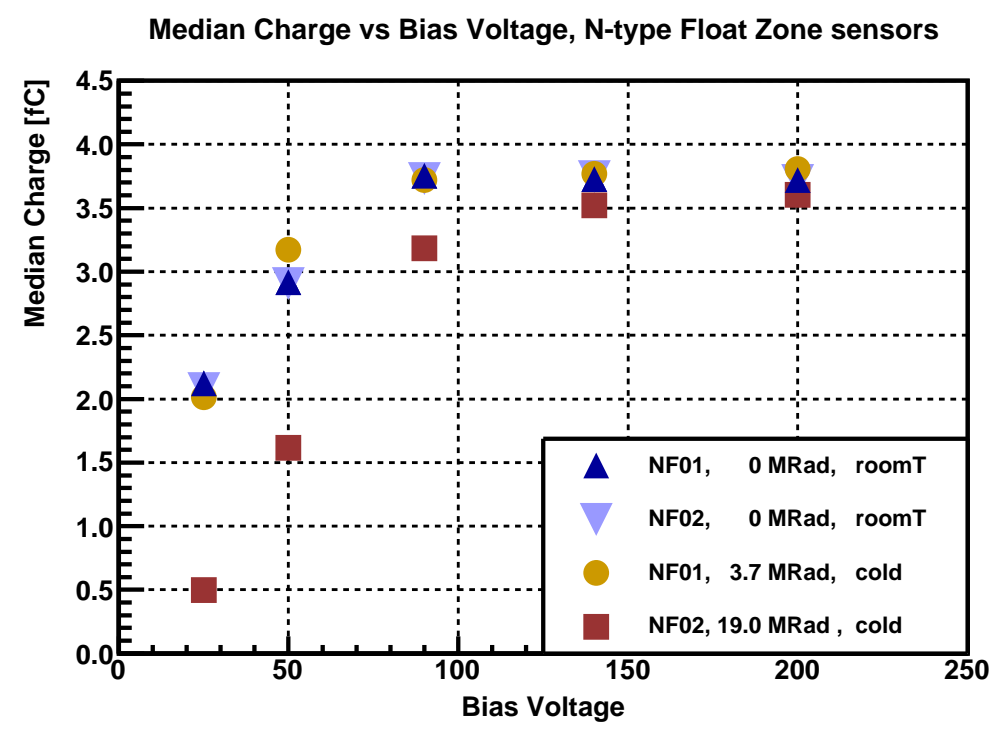

Figure 6: CC results for the NF01 (3.7 MRad dose) and NF02 (19.0 MRad dose) sensors.

[2] SID Collaboration, SiD Detailed Baseline Design, http://www.linearcollider.org/ILC/physics-detectors/Detectors/

Detailed-Baseline-Design, Chapter 5 (2013).

[3] J.R. Carter and R.G. Downing, 'Charged Particle Radiation Damage in Semiconductors: Effect of Low Energy Protons and High Energy Electrons on Silicon', Interim Technical Final Report, TRW Apce Technology Laboratories, May 1965.

[4] T. Noguchi and M. Uesugi, 'Electron Energy Dependence of Relative Damage Coefficients of Silicon Solar Cells for Space Use', Technical Design of the International PVSEC-5, Kyoto, Japan (1990).

[5] Geoffrey P. Summers et al., 'Damage Correlations in Semiconductors Exposed to Gamma, Electron, and Proton Radiations', IEEE Transactions on Nuclear Science 40, 1372 (1993).

[6] J.M. Rafi et al., 'Degradation of High-Resistivity Float Zone and Magnetic Czochralski n-type Silicon Detectors Subjected to 2-MeV Electron Irradiation', NIM A 604, 258 (2009). 
Table 5: Maximum median charge collection before and after irradiation. While the $\mathrm{NC} 02$ sensor was irradiated at a temperature of $5 \mathrm{C}$, it was accidentally annealed for approximately 5 hours at temperatures as high as $130 \mathrm{C}$.

\begin{tabular}{ccccc} 
Sensor & $\begin{array}{c}\text { Dose } \\
(\text { MRad })\end{array}$ & $\begin{array}{c}\text { Median CC Before } \\
\text { Irradiation (fC) }\end{array}$ & $\begin{array}{c}\text { Median CC After } \\
\text { Irradiation (fC) }\end{array}$ & $\begin{array}{c}\text { Fractional } \\
\text { Loss (\%) }\end{array}$ \\
\hline \hline PF05 & 5.1 & 3.70 & 3.43 & 7 \\
PF14 & 20 & 3.68 & 3.01 & 18 \\
PC08 & 20 & 3.51 & 3.09 & 12 \\
NF01 & 3.7 & 3.76 & 3.81 & 0 \\
NF02 & 19 & 3.75 & 3.60 & 4 \\
NC01 & 5.1 & 3.71 & 3.80 & 0 \\
NC10 & 18 & 3.76 & 3.74 & 1 \\
NC03 & 90 & 3.68 & 3.55 & 4 \\
NC02 & 220 & 3.69 & 3.06 & $17^{*}$ \\
\hline
\end{tabular}

[7] S. Dittongo et al., 'Radiation Hardness of Different Silicon Materials after High-Energy Electron Irradiation', NIM A 530, 110 (2004).

[8] S. Dittongo et al., 'Studies of Bulk Damage Induced in Different Silicon Materials by $900 \mathrm{Mev}$ Electron Irradiation', NIM A 546, 300 (2005).

[9] G. Casse et al., 'First Results on Charge Collection Efficiency of Heavily Irradiated Microstrip Sensors Fabricated on Oxygenated p-type Silicon', NIM A 518, 340 (2004).

[10] G. Casse, 'Radiation Hardness of p-type Silicon Detectors', NIM A 612, 464 (2010).

[11] The Electron Gamma Shower (EGS) Monte Carlo Program, http://rcwww.kek.jp/research/egs/.

[12] The specific device used was the REM Oxford Ltd. corporation's REM TOT601B device, http://www.radfet.com/index.html.

[13] Hartmut F.-W. Sadrozinski et al., 'The Particle Tracking Silicon Microscope PTSM', IEEE Transactions on Nuclear Science 51, 2032 (2004).

[14] K. Hara et al., 'Testing of bulk radiation damage of n-in-p silicon sensors for very high radiation environments', NIM A 636, S83 (2011). 